Ann. Biol. anim. Bioch. Biophys., 1977, 17 (1), 43-53.

\title{
Automatisation d'une méthode de dosage du fructose application à l'étude des oligosides du soja
}

par J. M. BESLE

Station de Recherches sur l'Elevage des Ruminants, INRA, Theix, Saint-Genes-Champanelle, 63110 Beaumont, France

Summary. Automatization of a sensitive fructose assay used in the study of soya oligosides.

We report the automatization of a sensitive specific assay of free fructose or that contained in various molecules. The manifold is shown in figure 1 . The operating condjtions are such that Beer Lambert's law is observed in a large concentration range (fig. 2a).

The Brij 35 improves baseline stability, increases sensitivity to low amounts, decreases it at high concentrations (fig. 2a, b) and changes the specificity (table 1). Using this method, $0.5 \mathrm{r} / \mathrm{ml}$ of fructose is assayed (fig. 3) with good reproducibility (coefficient of variation of 20 samples at $10 \gamma / \mathrm{ml}$ is $1.02 \mathrm{p} .100$ )

Reactivity of different sugars in aqueous or alcoholic solutions is measured in the conditions described (table 1). Fructose phosphoric esters react with the same sensitivity as fructose in aqueous solution. The effect of temperature on specificity and sensitiveness of the assay is shown in figure 4. Carbohydrates containing fructose (saccharose, raffinose, stachyose) are checked in table 2 to be sure that they are specifically assayed.

The method is used to specifically assay saccharose and the $\alpha$-galactosides (raffinose, stachyose) of various soya samples (oil meal, fermented oil meal, flour, fermented flour, soya feed) after separation by resin anion-exchange chromatography. These sugars are assayed at the same time using a total sugar evaluation method (sulfuric orcinol). Two methods for determinating fructose concentration in the column effluent are compared. One uses direct assay at the column exit at the same time as the total sugar is assayed. Oligoside concentration is obtained by comparison with the standard curves $S=f(C)(S=$ surface, $C=$ concentration) for each sugar. In the other method, the part of the effluent not used in total sugar assay is collected and the fructose is assayed on each carbohydratfraction. Multiplying the result by a constant measured for each sugar, oligoside concentration in the sample may be determined.

Results obtained by both fructose assay methods agree and they confirm those found by assaying the total sugar (table 3). Differences between the methods are non-significant $(P \leqslant 0.05)$. Thus, the peaks separated by the resin column contain no other carbohydrated which could interfere with the saccharose and the $\alpha$-galactosides. If they did, the unseparated carbohydrates would be evaluated by difference. The results on the carbohydrate composition of soya agree with those found by other authors. Fermentation eliminates a large part of the carbohydrates from the samples. The choice of a method for evaluating saccharose and $\alpha$-galactoside, using fructose, is discussed. The second is recommended because the curves $S=f(C)$ vary in time ; this may cause errors. 


\section{Introduction.}

L'étude de la digestion chez le veau, des glucides des plantes protéagineuses nous a conduit à utiliser une méthode de dosage du saccharose et des $\alpha$-galactosides qui soit à la fois rapide, spécifique et sensible. La chromatographie sur résine échangeuse d'ions (Besle, 1974) permet de séparer les di, tri, tétraholosides et parfois leurs homologues supérieurs, puis de les doser par une méthode colorimétrique des sucres totaux (orcinol sulfurique). Pour améliorer la spécificité du dosage et aussi pour éviter des erreurs dues à d'éventuelles interférences de glucides mal ou non séparés des $\alpha$-galactosides (raffinose, stachyose) et du saccharose, il nous a paru intéressant de déterminer ces glucides par une méthode dosant spécifiquement l'un des constituants qui leur est commun, c'est-à-dire le fructose. II nous a fallu trouver une méthode qui soit extrêmement sensible puisqu'en sortie de colonne, le fructose", se trouvait souvent à des concentrations de l'ordre de 0,5 à $1 \mathrm{r} / \mathrm{ml}$.

Les méthodes de dosage du fructose (constituant de différents glucides) se divisent en deux groupes. Dans le premier, le glucide est, au préalable, hydrolysé et le fructose est dosé par voie enzymatique (Bernt et Bergmeyer, 1974) ou par chromatographie (Wood et Cousins, 1966) ; dans le second, les glucides sont mis en présence d'un milieu très acide où le fructose (ainsi que les hexoses et les polymères, mais ceux-ci réagissent moins rapidement) se dégrade en hydroxyméthylfurfural qui donne une réaction colorée avec différents chromogènes.

Les méthodes du premier groupe sont peu sensibles et longues. Parmi celles du second groupe, les méthodes automatisées de Wood et Cousins (1966) ef de Galli ef Jeanmaire (1965) modifiée par Michel (non publiée), se sont révélées trop peu sensibles, la dernière ne permettant pas d'apprécier des quantités inférieures à $5 \gamma / \mathrm{ml}$. Aussi, nous avons cherché parmi les nombreuses méthodes manuelles décrites, la technique qui convenait le mieux au but proposé et avons tenté d'automatiser celle de Yaphe et Arsenault (1965) (modification du procédé de Roe, 1934).

\section{Matériel et méthodes.}

Les produits utilisés dérivent du soja, certains d'entre eux étant traiłés par fermentation (procédé Staron $\left({ }^{1}\right)$ ) : tourteau de soja, tourteau de soja fermenté, farine de soja, farine de soja fermenté, aliment soja ${ }^{2}$ ).

- Préparation des extraits glucidiques. L'extraction est effectuée à $40^{\circ} \mathrm{C}$ sous agitation par épuisement avec de l'eau distillée contenant $\mathrm{HgCl}_{2}$ à 1 p. 1000 . Ce produit inhibe l'action des enzymes glycolytiques et particulièrement des $\alpha$-galactosidases. Nous obtenons ainsi la totalité des glucides hydrosolubles.

On procède à la purification des extraits aqueux sur résines échangeuses d'ions selon la méthode de Montreuil (1957), modifiée par Besle ef Pitiot (1976).

(1) Procédé pour le traitement des tourteaux d'origine végétale : Brevet no 7107977.

(2) Tourteau de soja dépelliculé, ayant subi un traitement hydrothermique. 
- Dosage des sucres totaux ef analyse chromatographique. Les oligosides des extraits aqueux purifiés sont analysés par chromatographie d'échange d'ions (Besle, 1974). Ils sont dosés automatiquement en sortie de colonne par l'orcinol sulfurique.

\section{MÉTHODE PROPOSÉE.}

Nous avons automatisé la méthode de Yaphe et Arsenault (1965), après avoir étudié les points suivants : composition et stabilité des réactifs, conditions de mélange des réactifs (température ambiante : $20^{\circ} \pm 2{ }^{\circ} \mathrm{C}$; glace : $0^{\circ} \mathrm{C}$; obscurité), stabilité de la ligne de base, influence du Brij et de la température de réaction. A la suite de ce travail, nous proposons la méthode modifiée suivante :

- Principe. En milieu chlorhydrique concentré et à la température de $75^{\circ} \mathrm{C}$, le fructose est dégradé en hydroxyméthylfurfural qui réagit ensuite avec le résorcinol en présence d'acétal acétique, pour donner une coloration rose.

- Appareillage. Nous utilisons un auto-analyseur comprenant les éléments suivants : échantillonneuse, pompe péristaltique, bain-marie, colorimètre à trajet optique de $10 \mathrm{~mm}$, enregistreur linéaire à expanseur d'échelle $10 \mathrm{mV}$.

- Réactifs. - Brij 35, Touzart et Matignon ( $\left.{ }^{3}\right)$ : solution saturée dans l'eau à raison de $30 \mathrm{~g} / 100 \mathrm{ml}$.

- Acétal RP, Prolabo ( $\left.{ }^{4}\right)$. Ce produit doit être utilisé frais. Préparer une solution mère de $1 \mathrm{ml}$ étendue 25 fois dans de l'acide acétique RP (Prolabo), puis diluer celle-ci $(1,1 \mathrm{ml}$ dans $100 \mathrm{ml})$ dans l'acide acétique. Les solutions d'acétal se conservent 1 mois à l'obscurité.

- Solution acide : dans $80 \mathrm{ml}$ d'eau distillée, ajouter $150 \mathrm{ml}$ de la solution diluée d'acétal acétique ef $1950 \mathrm{ml}$ d'acide chlorhydrique fumant RP (Prolabo ( $\left.{ }^{4}\right)$ ). Ce réactif esi stable au moins 1 mois à température ambiante dans un flacon ambré hermétiquement fermé.

Ajouter extemporanément dans le volume nécessaire au dosage quotidien, la quantité de Brij 35 saturé donnant une solution de 0,1 à 0,7 p. 1000 , suivant la sensibilité de dosage désirée. Le Brij se dégrade lentement dans l'acide, ainsi il n'est pas bon de conserver la solution acide + Brij plus de deux jours. Nous appellerons "réactif acide », la solution acide + Brij.

- Résorcinol RP, Prolabo $\left.{ }^{4}\right)$ : solution à 1,5 p. 100 dans l'eau distillée. La conservation est d'au moins 1 mois à $4^{\circ} \mathrm{C}$.

- Schéma du montage. Le montage réalisé pour le dosage est décrit figure 1. La réaction s'effectue à $75^{\circ} \mathrm{C}$ et l'intensité de la coloration est mesurée à $555 \mathrm{~nm}$.

Le temps du prélèvement est de $1 \mathrm{~mm} 20 \mathrm{~s}$ et celui du rinçage de $2 \mathrm{mn}$. On peut adopter une cadence plus rapide, mais cela nuit à la sensibilité et à la reproductibilité du dosage.

(3) Touzart et Matignon, 3, rue Amyot, 75005 PARIS.

(4) Prolabo, 12, rue Pelée, 75011 PARIS. 
Il est nécessaire d'éliminer la bulle qui se forme au moment du prélèvement de l'échantillon car elle produit un pic parasite. Le montage esł réalisé avec des tubes en verre, reliés par des manchons en acidflex. La distribution des bulles de segmentation doit être très régulière pour avoir une ligne de base stable. Le colorimètre avec expanseur d'échelle permet de sélectionner la gamme d'étalonnage suivant la concentration de l'échantillon : 0,5 à $5 \gamma / \mathrm{ml}$ jusqu'à 5 à $40 \gamma / \mathrm{ml}$.

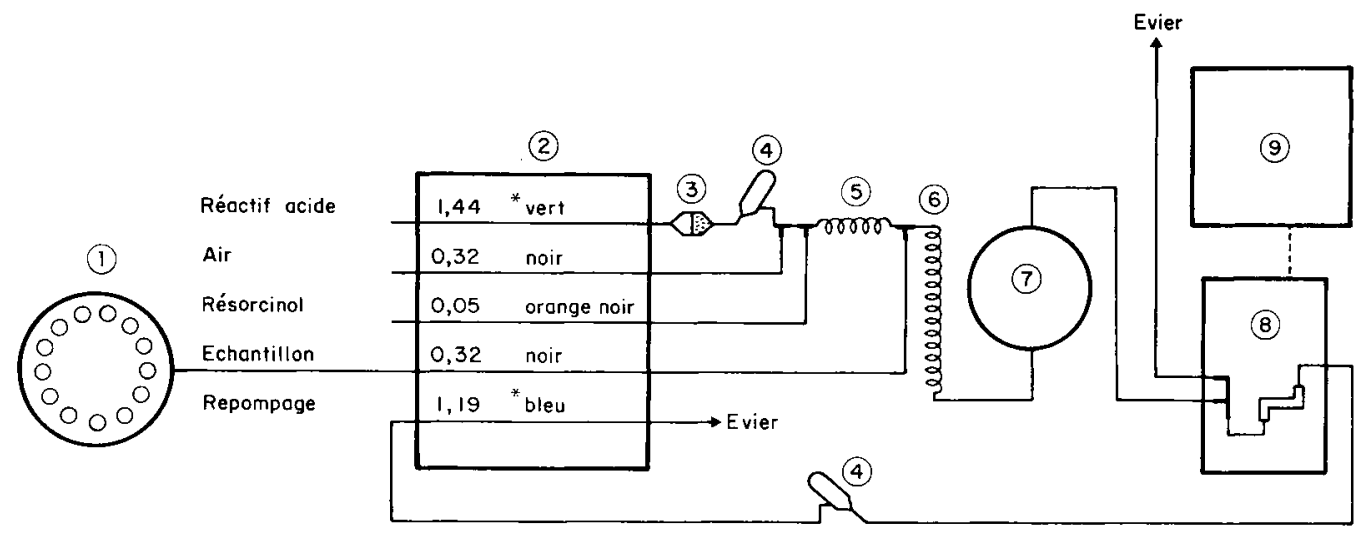

FIG. 1. - Circuit de dosage en continu du fructose

(1) Echantillonneuse Hook et Tucker A 40, prélèvement $1 \mathrm{mn} 20 \mathrm{~s}$, rinçage $2 \mathrm{mn}$.

(2) Pompe péristaltique Technicon.

(3) Filtre en laine de verre.

(4) Chambre antireflux en verre.

(5) Petiłe bobine de mélange.

(6) Grande bobine de mélange.

(7) Bain-marie $75^{\circ} \mathrm{C}$, serpentin $\varnothing$ int. $1,6 \mathrm{~mm}$.

(8) Colorimètre Vitatron $555 \mathrm{~nm}$.

(9) Enregistreur Vitatron à expanseur d'échelle.

* Tubes en acidflex.

Nous avons observé qu'au bout d'un certain temps, un dépôł de chromogène rose se forme sur les tubes et finit par gêner le dosage en produisant des petites particules. Ce dépôt doit être éliminé préiodiquement (chaque semaine) à l'aide d'une solution de soude $3 \mathrm{~N}$ ou par du détergent de laboratoire (DDN 50) ${ }^{5}$ ).

Le passage d'acide chlorhydrique concentré à travers le tube en acidflex le distend, ce qui modifie la sensibilité du dosage avec le temps. Elle diminue régulièrement tout au long de la journée. De ce fait, il faut intercaler régulièrement (tous les 20 échantillons) une solution étalon de fructose pour tenir compte des variations de sensibilité.

Dans les conditions opératoires que nous préconisons, le coefficient de variation de 20 échantillons de $10 \mathrm{\gamma} / \mathrm{ml}$ de fructose est de 1,02 p. 100. Enfin, le fructose peut être dosé en solution alcoolique à $80^{\circ} \mathrm{GL}$, la sensibilité étant comparable à celle en solution aqueuse.

(5) Hydromesures, 28 bis, rue A.-Cayron, 92600 ASNIĖRES. 


\section{Résultats.}

\section{VALIDITÉ ET LIMITES DE LA MÉTHODE.}

- Observation de la loi de Beer Lambert. La quantité d'acétal a été déterminée de manière que la sensibilité soit maximale et que la loi de Beer Lambert soit observée sur une large gamme de concentration (voir fig. 2a). Ce résultat est favorisé en outre par le prémélange résorcinol-réactif acide avant l'arrivée de l'échantillon, sur le circuit de dosage.

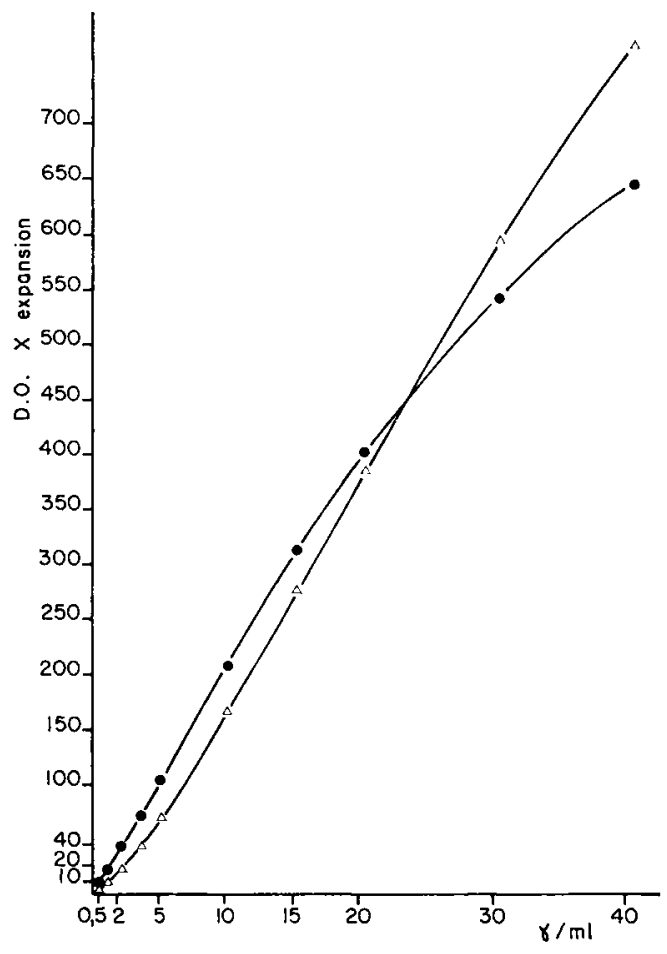

$\triangle$ Sans Brij. Avec Brij 0,7 p. 1000 .

FIG. 2a. - Influence du Brij sur la forme de la courbe étalon du fructose

Température de réaction : $75^{\circ} \mathrm{C}$.

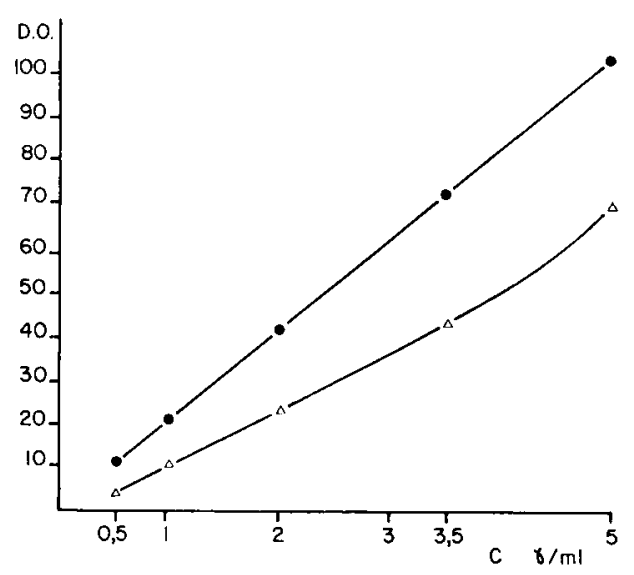

$\triangle$ Brij 0,1 p. 1000 . Brij 0,7 p. 1000.

FIG. 2b. - Influence de la concentration en Brij sur la forme de la courbe étalon du fructose Mesure à la sensibilité maximale de l'enregistreur. Température de la réaction : $75^{\circ} \mathrm{C}$.

- Influence du Brij. Afin d'améliorer la qualité de la ligne de base, nous avons rajouté à la solution acide, du Brij 35 . Ce produit, toutefois, interfère dans la réaction, il augmente la sensibilité aux faibles concentrations en fructose mais la diminue aux fortes valeurs. De ce fait, la loi de Beer Lambert n'est pas observée dans le même intervalle de concentration (fig. $2 a$ et $2 b$ ). Il convient de ne pas ajouter plus de 0,7 p. 1000 
de Brij, car au-delà de cette concentration, il ne produit plus de modifications notables de la sensibilité et nuit à la qualité de la ligne de base car il réagit avec les réactifs seuls. Aussi, lorsque la sensibilité maximale n'est pas désirée, nous conseillons de n'employer qu'une solution à 0,1 p. 1000 . La figure 3 montre la gamme étalon de fructose pour une concentration en Brij 35 de 0,7 p. 1000 cbtenue avec la sensibilité maximale de l'enregistreur. Nous pouvons doser $0,5 \gamma / \mathrm{ml}$.

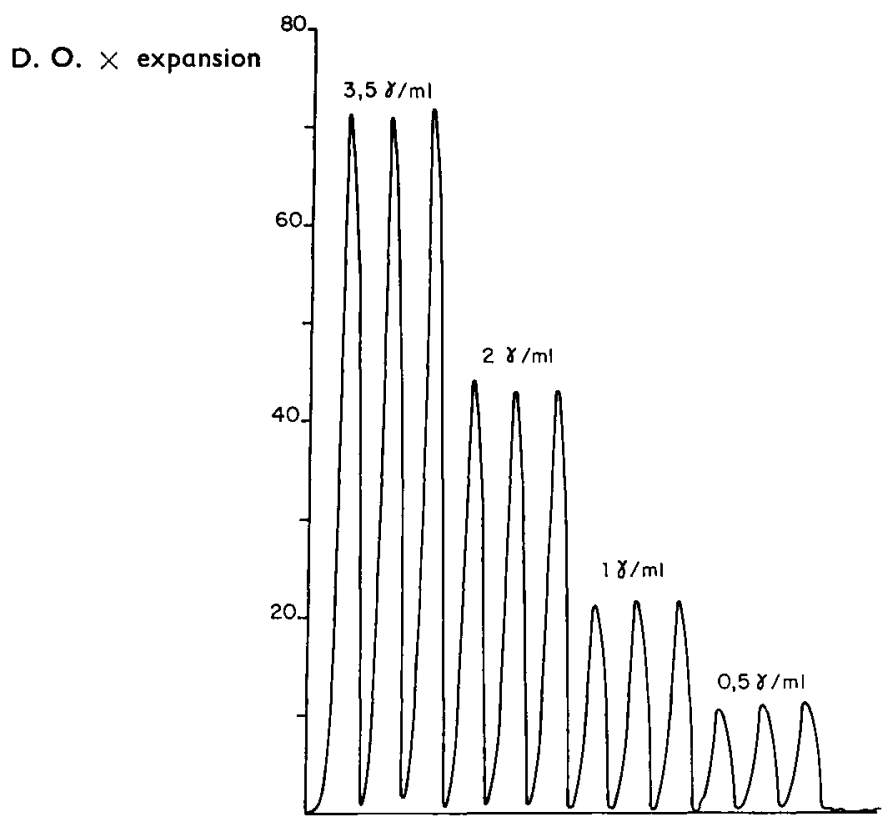

FIG. 3. - Gamme étalon du fructose : Mesure à la sensibilité maximale de l'enregistreur Concentration en Brij : 0,7 p. 1000 . Température de réaction : $75^{\circ} \mathrm{C}$.

- Spécificité de la réaction, influence de la température. La spécificité de la réaction provient du fait que le fructose, grâce à sa forme furane, est plus rapidement dégradé en dérivé du furfural que les autres glucides. Elle est donc améliorée en jouant sur la vitesse de réaction ou sur la température à temps égal.

Le temps de passage dans une bobine de bain-marie (diamètre interne $1,6 \mathrm{~mm}$ ) est de $12 \mathrm{mn}$ pour ce montage. II correspond à peu près à celui préconisé par Yaphe et Arsenault (1956) $(10 \mathrm{mn})$. Nous avons donc éfudié l'influence de la température et du Brij sur la sensibilité et la spécificité du dosage.

La figure 4 montre la variation de la sensibilité du dosage ef de la réactivité de différents sucres en fonction de la température. Nous opterons pour la température de $75^{\circ} \mathrm{C}$ où la sensibilité afteint un maximum et où les interférences des autres sucres sont moindres. Le sorbose qui est un cétose, a une réactivité de 70 p. 100 par rapportà celle du fructose. 
Le Brij modifie la réactivité (łabl. 1), le rhamnose inferfère particulièrement plus, alors que les autres sucres réagissent de façon comparable ou plutôt moins. En milieu alcoolique à $80^{\circ} \mathrm{GL}$, la spécificité est peu modifiée. En solution aqueuse, les esters phosphoriques du fructose sont dosés avec une sensibilité équivalente à ce dernier, surtout aux fortes concentrations en Brij. Par contre, en solution éthanolique, leur réaction est très différente, particulièrement pour le fructose 1, 6-diphosphate (łabl. 1).

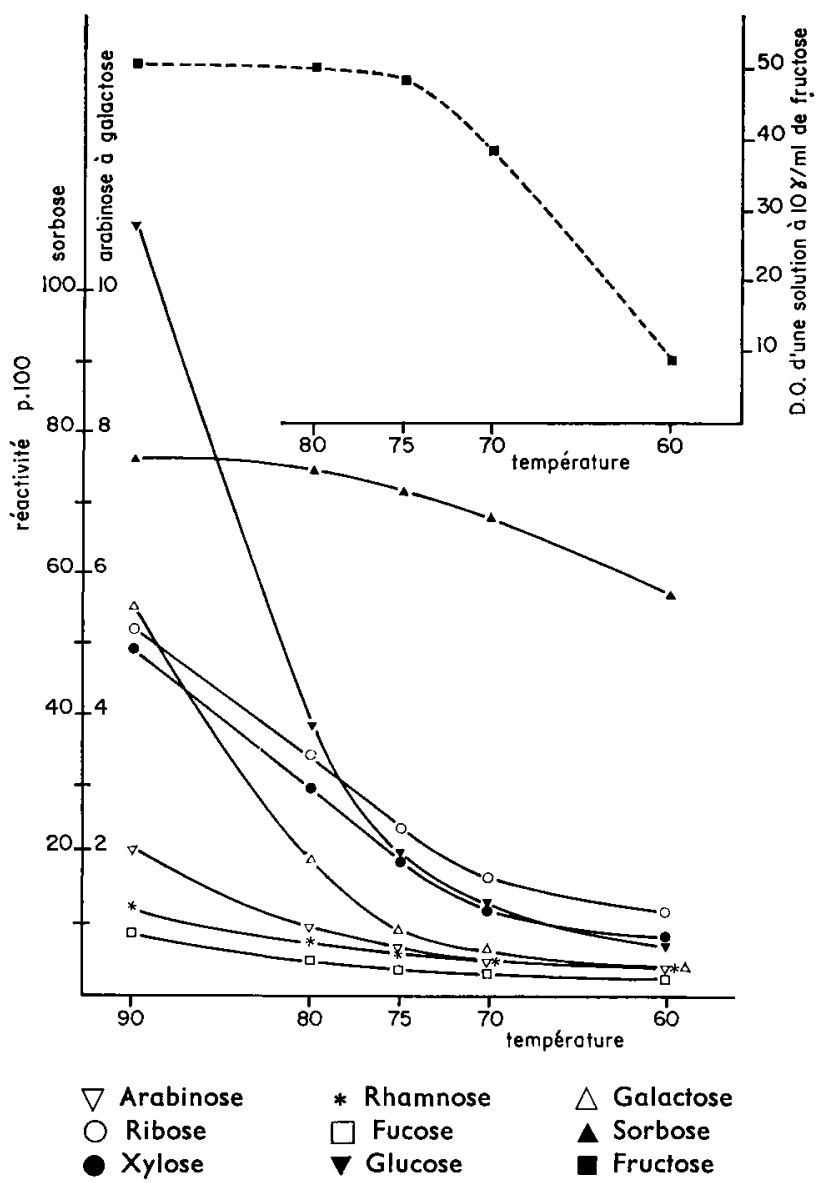

FIG. 4. - Influence de la fempérature de réaction sur la spécificité du dosage

La réactivité de chaque glucide est évaluée pour une valeur 100 du fructose à poids égal. Concentration en Brij : 0,1 p. 1000.

La faible interférence du glucose et du galactose, permet de doser de façon spécifique le fructose libre ou lié dans les oligosides. Le dosage du saccharose, du raffinose ef du stachyose conduit à l'obtention d'une courbe étalon linéaire dans le même intervalle de concentration que le fructose qui les compose. En outre, ce dosage permet de retrouver la quantité pesée de ces sucres avec une bonne précision, à diverses 
concentrations, compte tenu de leur degré d'hydratation (évalué suivant les indications du fabricant, mais pouvant varier avec les conditions de stockage) (tabl. 2).

\section{TABLEAU 1}

Réactivité à $75^{\circ} \mathrm{C}$ des glucides en solution aqueuse et alcoolique Influence du Brij dans le cas des solutions aqueuses

\begin{tabular}{|c|c|c|c|}
\hline & \multicolumn{2}{|c|}{ Solution aqueuse } & \multirow{2}{*}{$\begin{array}{c}\begin{array}{c}\text { Solution } \\
\text { éthanol d̀ } 80^{\circ}\end{array} \\
\text { Brij } 0,1 \text { p. } 1000 \\
\text { Réactivité p. } 100\left({ }^{1}\right)\end{array}$} \\
\hline & $\begin{array}{l}\text { Brij 0,1 p. } 1000 \\
\text { Réactivité P. } 100\left({ }^{1}\right)\end{array}$ & $\begin{array}{l}\text { Brij } 0,7 \text { p. } 1000 \\
\text { Réactivité p. } 100\left({ }^{1}\right)\end{array}$ & \\
\hline 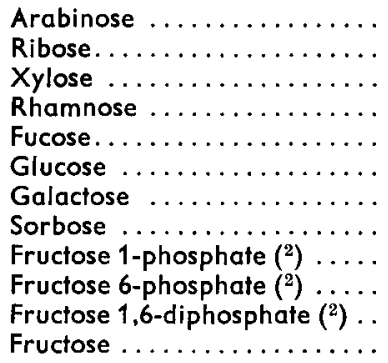 & $\begin{array}{l}0,58 \\
2,26 \\
1,83 \\
0,59 \\
0,31 \\
1,95 \\
0,87 \\
68,0 \\
94,6 \\
95,6 \\
83,9 \\
100\end{array}$ & $\begin{array}{c}0,28 \\
1,30 \\
0,87 \\
1,47 \\
0,46 \\
1,65 \\
0,67 \\
68,0 \\
99,2 \\
98,1 \\
93,3 \\
100\end{array}$ & $\begin{array}{c}0,52 \\
1,60 \\
1,45 \\
0,65 \\
0,34 \\
1,80 \\
0,83 \\
87,0 \\
83,6 \\
95,7 \\
64,8 \\
100\end{array}$ \\
\hline
\end{tabular}

(1) Réactivité du glucide pour une valeur 100 du fructose, ramené à un poids égal de $10 \mathrm{\gamma} / \mathrm{ml}$.

( ${ }^{2}$ La concentration est ramenée en équivalent fructose pour le calcul de la réactivité_de_ces molécules.

Nous avons vérifié en outre que le borate de sodium $(0,6 \mathrm{M}$ par litre, $\mathrm{pH} 9,5)$ et le chlorure de sodium (0,2 M par litre) n'interfèrent pas. Ceci est très important car cette méthode peut alors être utilisée pour doser les sucres dans l'effluent de la colonne de chromatographie d'échange d'ions, qui contient ces deux sels à des concentrations voisines de celles que nous avons étudiées.

TABLEAU 2

Taux de récupération de glucides contenant du fructose

(Dosage à $75^{\circ} \mathrm{C}$ avec Brij à 0,1 p. 1000 )

\begin{tabular}{cccccc}
\hline \multicolumn{2}{c}{ Saccharose } & \multicolumn{2}{c}{ Raffinose } & \multicolumn{2}{c}{ Stachyose } \\
\hline $\begin{array}{c}\text { Concentration } \\
(\gamma / \mathrm{ml})\end{array}$ & $\begin{array}{c}\text { Taux }\left({ }^{1}\right) \\
(\mathrm{p} .100)\end{array}$ & $\begin{array}{c}\text { Concentration } \\
(\gamma / \mathrm{ml})\end{array}$ & $\begin{array}{c}\text { Taux }\left({ }^{1}\right) \\
(\mathrm{p} .100)\end{array}$ & $\begin{array}{c}\text { Concentration } \\
(\gamma / \mathrm{ml})\end{array}$ & $\begin{array}{c}\text { Taux }\left({ }^{1}\right) \\
(\mathrm{p} .100)\end{array}$ \\
\hline 10 & 97 & 15 & 103 & 20 & 104,5 \\
20 & 100 & 30 & 99 & 40 & 104,5 \\
40 & 102 & 30 & 101 & 80 & 102,0 \\
\hline $\begin{array}{c}\text { Taux moyen } \\
(\mathrm{p} .100)\end{array}$ & 99,7 & & 101 & & 103,7 \\
\hline
\end{tabular}

(1) Taux de récupération calculé par rapport à une gamme étalon de fructose. 
La très grande sensibilité et la spécificité satisfaisante de la méthode proposée lui confèrent une large application. II faut toutefois noter que si le fructose se trouve en petite quantité par rapport à d'autres sucres, il est nécessaire d'utiliser un facteur correctif tenant compte de la réactivité de ceux-ci.

\section{APPLICATION AU DOSAGE DES OLIGOSIDES DU SOJA.}

Nous avons utilisé la méthode proposée pour vérifier la pureté chimique des pics obtenus par chromatographie échangeuse d'ions des oligosides du soja : saccharose, raffinose, stachyose qui contiennent tous du fructose.

Afin d'évaluer ce dernier, deux modes opératoires sont possibles. L'un consiste à le doser directement en sortie de colonne, parallèlement aux sucres tołaux, la réaction se faisant à la même température $\left(85^{\circ} \mathrm{C}\right.$ ) avec 2 colorimètres enregistreurs. L'autre est de le doser ultérieurement dans les fractions glucidiques d'une partie de l'effluent collecté sur fractionneuse, le restant servant au dosage en continu des sucres totaux.

\section{TABLEAU 3}

Analyse des glucides par chromatographie échangeuse d'ions. Vérification de la pureté chimique des pics (Concentration * évaluée en p. 100 de la matière sèche)

\begin{tabular}{|c|c|c|c|c|c|c|c|c|c|c|c|c|c|c|c|}
\hline \multirow{2}{*}{$\begin{array}{c}\text { Echantillons } \\
\text { Méthode }\end{array}$} & \multicolumn{3}{|c|}{$\begin{array}{l}\text { Tourteau } \\
\text { de soja }\end{array}$} & \multicolumn{3}{|c|}{$\begin{array}{l}\text { Farine } \\
\text { de soja }\end{array}$} & \multicolumn{3}{|c|}{ Aliment soja } & \multicolumn{3}{|c|}{$\begin{array}{l}\text { Tourteau de } \\
\text { soja fermenté }\end{array}$} & \multicolumn{3}{|c|}{$\begin{array}{l}\text { Farine de soja } \\
\text { fermentée }\end{array}$} \\
\hline & 1 & 2 & 3 & 1 & 2 & 3 & 1 & 2 & 3 & 1 & 2 & 3 & 1 & 2 & 3 \\
\hline $\begin{array}{l}\text { Saccharose } \\
\text { Raffinose... } \\
\text { Stachyose.. }\end{array}$ & $\begin{array}{l}6,27 \\
0,80 \\
3,99\end{array} \mid$ & $\mid \begin{array}{l}6,75 \\
0,87 \\
3,85\end{array}$ & \begin{tabular}{|l|}
6,57 \\
0,87 \\
3,85
\end{tabular} & $\begin{array}{l}8,03 \\
0,84 \\
2,66\end{array}$ & $\begin{array}{l}7,75 \\
0,93 \\
2,49\end{array}$ & $\left|\begin{array}{l}7,52 \\
0,91 \\
2,87\end{array}\right|$ & \begin{tabular}{|l|}
6,98 \\
1,02 \\
4,89
\end{tabular} & $\begin{array}{l}6,83 \\
0,94 \\
4,75\end{array}$ & $\begin{array}{l}6,73 \\
1,02 \\
4,50\end{array}$ & $\begin{array}{l}0,013 \\
0,253\end{array}$ & 0,243 & 0,249 & $\left|\begin{array}{l}0,014 \\
0, \overline{330}\end{array}\right|$ & 0,342 & 0,323 \\
\hline
\end{tabular}

1. Dosage en sortie de colonne par la réaction à l'orcinol sulfurique (sucres totaux). Evaluation quantitative à partir de la surface des pics.

2. Dosage du fructose en sortie de colonne. Evaluation quantitative à partir de la surface des pics.

3. Dosage du fructose sur les fractions collectées. Evaluation quantitative à partir des coefficients calculés pour chaque sucre.

* Ces résultats sont la moyenne de trois essais par échantillon.

L'examen du tableau 3 montre que les résultats obtenus par les 2 procédés de dosage du fructose concordent entre eux et avec ceux obtenus par le dosage des sucres totaux. L'analyse de variance à 2 facteurs (méthode, échantillons) indique que les différences entre les méthodes ne sont pas significatives au seuil de probabilité 5 p. 100. Nous démontrons ainsi d'une part, l'équivalence au niveau des résultats des 2 procédés d'évaluation du fructose, ef d'autre part la pureté chimique des pics de saccharose, raffinose, stachyose. Nous voyons en outre que les produits fermentés ne contiennent plus qu'un peu de stachyose et des traces de saccharose. 


\section{Discussion.}

\section{INTÉRET DE LA MÉTHODE PROPOSÉE.}

Toutes les fois qu'une grande sensibilité, jointe à une bonne spécificité, seront nécessaires, cette méthode trouvera son application. Le fructose peut être dosé en solution aqueuse comme en milieu éthanolique, il peut être libre ou contenu dans diverses molécules glucidiques ou hétérosidiques, ses esters phosphoriques réagissent avec une sensibilité équivalente en solution aqueuse.

Utilisée pour le dosage des glucides du soja, nous avons pu démontrer, grâce à cette méthode, l'absence d'interférences dans les pics de l'effluent de la colonne et pourrons évaluer par différence, lors de l'expérience sur animaux, les glucides non séparés (par exemple le couple lactose-stachyose de l'alimenł lait de soja).

La composition des échantillons de soja non fermentés s'est révélée comparable à celle trouvée par d'autres auteurs (Tanret, 1913 ; Daubert, 1950 ; Täufel, Steinbach et Vogel, 1960 ; Kawamura, Tada et Narasaki, 1966 ; Delente et Ladenburg, 1972). Nous avons aussi vérifié que la fermentation permet d'éliminer la majeure partie des oligosides.

\section{ÉVALUATION DU SACCHAROSE ET DES $\alpha$-GALACTOSIDES DU SOJA.}

Dans le cas spécial du dosage du saccharose et des $\alpha$-galactosides du soja (ou d'autres produits pouvant en contenir) par chromatographie échangeuse d'ions, le fructose peut être évalué, soit directement en sortie de colonne, soit dans les fractions recueillies avec un collecteur.

Dans le premier cas, si le développement de la réaction colorimétrique ne pose pas de problèmes, il en est différemment lorsque l'on veut évaluer les glucides quantitativement. En effet, la courbe étalon du fructose ne suit pas la loi de Beer Lambert pour les faibles concentrations en ce sucre. II ne suffit pas de multiplier simplement la surface des pics par une constante pour avoir la concentration en glucides. II est nécessaire de tracer une courbe $S=f(C)(S=$ surface, $C=$ concentration) avec des solutions étalons de glucides contenant du fructose ef de procéder par comparaison pour les échantillons inconnus. Cette surface est corrigée pour un volume d'échantillon de $0,1 \mathrm{ml}$ et par rapport à une solution standard externe de fructose qui tient compte de la variabilité due au montage permettant le dosage en sortie de colonne.

$$
\mathrm{S}=\frac{\mathrm{h} \times \mathrm{a} \times \text { échelle d'expansion } \times \mathrm{SE} \text { ref }}{\mathrm{Vi} \times 10} \times \mathrm{SE} \mathrm{0,5}
$$

$\mathbf{h}=$ Hauteur du pic en $\mathrm{mm}$.

$a=$ Largeur à mi-hauteur en $\mathrm{mm}$.

Echelle d'expansion : intervalle de densité optique couvert par l'enregistreur.

$V i=$ Volume d'échantillon déposé en haut de colonne $(\mathrm{ml})$.

$S E$ ref $=$ Standard externe de référence. Densité optique mesurée une fois pour toutes

d'une solution de fructose à $10 \mathrm{\gamma} / \mathrm{ml}$ pour l'échelle 0-0,5.

SE $0,5=$ Standard externe pour l'échelle 0-0,5. Valeur en densité optique obtenue avant chaque chromatographie. 
Cet éłalonnage est long puisqu'il demande au moins cinq chromatogrammes et demande à être refait assez souvent du fait d'une grande variabilité du coefficient de réponse. La variation de débit avec le temps, du tuyau en acidflex véhiculant le réactif acide, modifie la sensibilité du dosage et le temps nécessaire pour traverser l'autoanalyseur. Ceci nuit à la précision de la méthode.

Dans le second cas, il faut tenir compte du fait qu'une partie des sucres est dégradée dans la colonne et calculer un coefficient qui permet de refrouver la quantité déposée en haut de colonne à partir de la quantité de fructose dosée. D'après nos travaux antérieurs sur la chromatographie échangeuse d'ions (Besle, 1974), nous pouvons penser que ce coefficient est constant et ne dépend pas de la concentration en glucides. Toutefois, nous l'avons calculé pour des concentrations voisines de celles de nos échantillons. Cette méthode ne peut être employée que lorsque les pics sont bien séparés. La fraction d'effluent qui est analysée en continu à l'orcinol sulfurique permet d'obtenir un chromatogramme où l'on repère les fractions intéressantes. Ce procédé ne nécessite qu'un collecteur de fraction au lieu d'un doublement du système colorimètre-enregistreur. Pour avoir pratiqué les deux procédés, nous recommandons le second plutôt que le premier car nous avons observé une grande variabilité des courbes d'étalonnage $S=f(C)$, ce qui est cause d'erreur.

Accepté en juillet 1976.

\section{Références}

BERNT E., BERGMEYER H. U., 1974. Methods for determination of metabolites : D-Fructose, in H. U. BERGMEYER Methods of Enzymatic Analysis., Verlag Chemie Weinheim, Acad. Press. Inc. New York and London, Vol 3, 1304-1307.

BESLE J. M., 1974. Séparation des oses, des di- et triholosides par chromatographie d'échange d'ions. Ann. Biol. anim. Bioch. Biophys., 14, 545-573.

BESLE J. M., PITIOT M., 1976. Extraction ef purification des glucides : application à divers aliments dérivés du soja. Ann. Biol. anim. Bioch. Biophys., 16, sous presse.

DAUBERT B. F., 1950. Other constituents of the soybean : carbohydrates. In K. S. Marten Soybean and soybean products, Vol. 1, 371-381.

DELENTE J., LADENBURG K., 1972. Quantifative determination of the oligosaccharides in defatted soybean meal by gas-liquid chromatography. J. Fd. Sci., 37, 372-374.

GALLI A., JEANMAIRE J., 1965. Dosage automatique du fructose par la réaction à l'acide thiobarbiturique. Ann. Biol. Clin., 23, 925-930.

KAWAMURA S., TADA M., NARASAKI T., 1966. Sugars of the cotyledon, hull and hypocotyl of soybeans. J. Jap. Soc. Food Nutrition, 19, 268-275.

MICHEL M. C., 1975. Communication personnelle.

MONTREUIL J., 1957. Glycoprotéides. Bull. Soc. Chim. Biol., 39, Suppl. III, 1-92.

ROE J. H., 1934. A colorimetric method for the determination of fructose in blood and urine. J. Biol. Chem., 107, 15-22.

TANRET G., 1913. Sur la présence du stachyose dans le haricot et les graisses de quelques autres légumineuses. Bull. Soc. Chim. France, 13, 176-182.

TÄUFEL K., STEINBACH K. S., VOGEL E., 1960. Mono- und oligosaccharide einiger Leguminosensamen sowie ihr Verhalten bei Lagerung und Keimung. Z. Lebensmittelsunters u. Forsch., 112, $31-40$.

WOOD P. J., COUSINS A. R., 1966. The analysis of sucrose, fructose and glucose in tobacco. Automation in analytical chemistry. E. KAWERAU, European Technicon Symposium, Paris. Mediad. Inc. New York, 2, 97-102.

YAPHE W., ARSENAULT G. P., 1965. Improved resorcinol reagent for the determination of fructose, and of 3,6 anhydrogalactose in polysaccharides. Anal. Biochem., 13, 143-148. 